\title{
Premotor Neurons Encode Torsional Eye Velocity during Smooth-Pursuit Eye Movements
}

\author{
Dora E. Angelaki and J. David Dickman \\ Department of Neurobiology, Washington University School of Medicine, and Hearing Research Department, Central Institute for the Deaf, St. Louis, \\ Missouri 63110
}

\begin{abstract}
Responses to horizontal and vertical ocular pursuit and head and body rotation in multiple planes were recorded in eye movementsensitive neurons in the rostral vestibular nuclei (VN) of two rhesus monkeys. When tested during pursuit through primary eye position, the majority of the cells preferred either horizontal or vertical target motion. During pursuit of targets that moved horizontally at different vertical eccentricities or vertically at different horizontal eccentricities, eye angular velocity has been shown to include a torsional component the amplitude of which is proportional to half the gaze angle ("half-angle rule" of Listing's law). Approximately half of the neurons, the majority of which were characterized as "vertical" during pursuit through primary position, exhibited significant changes in their response gain and/or phase as a function of gaze eccentricity during pursuit, as if they were also sensitive to torsional eye velocity. Multiple linear regression analysis revealed a significant contribution of torsional eye movement sensitivity to the responsiveness of the cells. These findings suggest that many VN neurons encode three-dimensional angular velocity, rather than the two-dimensional derivative of eye position, during smooth-pursuit eye movements. Although no clear clustering of pursuit preferred-direction vectors along the semicircular canal axes was observed, the sensitivity of $\mathrm{VN}$ neurons to torsional eye movements might reflect a preservation of similar premotor coding of visual and vestibular-driven slow eye movements for both lateral-eyed and foveate species.
\end{abstract}

Key words: eye movement; vestibulo-ocular; vergence; kinematics; torsion; smooth pursuit; coordinate frame; three-dimensional; sensorimotor

\section{Introduction}

How the brain controls ocular torsion is a matter of considerable debate. The controversy has centered primarily on issues of neural versus mechanical contributions to the control of torsion for saccadic eye movements (Miller, 1989; Schnabolk and Raphan, 1994; Tweed et al., 1994; Tweed, 1997; Quaia and Optican, 1998; Raphan, 1998; Demer et al., 2000). All saccades that originate from primary position are associated with rotation axes that are confined to a single horizontal-vertical plane, referred to as "Listing's plane" (Helmholtz, 1867; Tweed and Vilis, 1987, 1990; Haslwanter et al., 1992; Tweed et al., 1992). However, when saccades are generated from nonprimary eye positions (i.e., tertiary saccades), the angular velocity axis of the eye does not remain confined to this plane but rather deviates toward the direction of gaze by approximately half as much ("half-angle rule") (Tweed and Vilis, 1987, 1990). When expressed in a head-fixed coordinate system, a torsional component the amplitude of which is related linearly to eye position is added to eye velocity during saccades that do not originate from primary position. In contrast, eye orientation and the derivative of eye orientation remain confined to the horizontal-vertical plane. This occurs because the mathematics of rotations do not follow simple vector algebra, e.g., angular velocity is not equal to the derivative of eye orienta-

Received Dec. 4, 2002; revised Jan. 22, 2003; accepted Jan. 24, 2003.

This work was supported by National Institutes of Health Grants EY-12814 and DC-04260, National Aeronautics and Space Administration Grant NAG2-1493, and the McDonnell Foundation for higher brain function.

Correspondence should be addressed to Dr. Dora Angelaki, Department of Anatomy and Neurobiology, Box 8108, Washington University School of Medicine, 660 South Euclid Avenue, St. Louis, M0 63110. E-mail: angelaki@thalamus.wustl.edu.

Copyright $\odot 2003$ Society for Neuroscience $\quad 0270-6474 / 03 / 232971-09 \$ 15.00 / 0$ tion, unless the eye is in primary position (Tweed and Vilis, 1987, 1990; Haslwanter, 1995).

This computational difficulty in extending knowledge acquired during horizontal-vertical oculomotor tasks to real, three-dimensional (3D) rotations of the eye was first addressed in the late 1980s by Tweed and Vilis $(1987,1990)$. Specifically, because angular velocity is not the derivative of eye orientation in $3 \mathrm{D}$, traditional oculomotor concepts that were well established for the horizontal system, like that of the velocity-to-position neural integrator (Skavenski and Robinson, 1973; Robinson, 1981; Cannon and Robinson, 1987), needed to be reevaluated. Under the assumptions that (1) the direction of action of extraocular muscles remains head-fixed and (2) premotor and motor neurons encode the 3D angular velocity of the eye, extension of the neural integration concept in $3 \mathrm{D}$ would require the incorporation of nonlinear (multiplicative) mathematical operations (Tweed and Vilis, 1987, 1990). Recently, both of these hypotheses have been challenged. First, mobile, soft-tissue sheaths or "pulleys" in the orbit have been proposed to influence the pulling direction of the extraocular muscles (Miller, 1989; Demer et al., 2000; Kono et al., 2002). In fact, a theoretical study by Quaia and Optican (1998) has shown that appropriately placed pulleys can both generate physiologically realistic saccades and implement the half-angle rule without a need to calculate a "neural torsional signal" as proposed by Tweed and Vilis (1987, 1990). Second, Henn and colleagues failed to find evidence for a clear neural representation of $3 \mathrm{D}$ angular velocity in the premotor pathway of saccadic eye movements (van Opstal et al., 1991, 1996; Hepp et al., 1993, 1999; Scherberger et al., 2001).

If the two-dimensional (2D) derivative of eye orientation, in- 
stead of 3D angular velocity, is encoded neurally, the neural integration process could be linear (and a direct extension of Robinson's one-dimensional integrator to 3D), but a kinematically and dynamically appropriate oculomotor behavior would need to rely on the eye plant itself. Although at present, this latter hypothesis appears to be favorable for saccadic eye movements, several issues remain unresolved. The most important problem is related to the rotational vestibulo-ocular reflex (VOR), in which, in contrast to visually guided eye movements, the vestibular sensory signal encodes 3D angular velocity (and not the derivative of eye orientation). Thus, "inverse" multiplicative calculations from those proposed originally by Tweed and Vilis for saccades are required in the VOR pathway, if it is to be compatible with an eye plant with pulleys (Smith and Crawford, 1998; Misslisch and Tweed, 2001).

Surprisingly, these issues have never been addressed for smooth-pursuit eye movements. Specifically, similar to saccades, premotor pursuit neural activity could be restricted to a coding of the $2 \mathrm{D}$ (horizontal-vertical) derivative of eye orientation rather than $3 \mathrm{D}$ angular eye velocity. Such a hypothesis would be in line with the 2D nature of both visual sensory signals and motor output (because pursuit, like saccades, follows Listing's law) (Haslwanter et al., 1991; Tweed et al., 1992). Alternatively, one could speculate that $3 \mathrm{D}$ coding of pursuit velocity might be advantageous if pursuit-VOR interactions were to share a common, semicircular canal (SCC)defined coordinate system. The premotor coding for pursuit would then be similar to the common coordinate system used for optokinetic-VOR convergence, as was shown to exist in birds and rabbits (Graf et al., 1988; Tan et al., 1993; Wylie and Frost, 1993; van der Steen et al., 1994). An SCC coordinate system hypothesis for pursuit could be supported by at least two experimental findings. First, pursuit and the VOR are thought to share common premotor neurons (Scudder and Fuchs, 1992; Cullen and McCrea, 1993; Lisberger et al., 1994). Second, the presence of strong torsional nystagmus during vertical pursuit in some patients with cerebellar deficits could be explained by assuming that premotor pursuit signals are coded in an SCC coordinate system (FitzGibbon et al., 1996). However, this hypothesis has never before been investigated in neural responses.

Similar to saccades, a torsional eye velocity component is always elicited during horizontal and vertical pursuit in eccentric eye orientations. Thus, in the present study, we used smooth-pursuit eye movements at different eccentric target positions to examine whether premotor neural pursuit activities exhibit evidence for 3D (torsional) ocular sensitivity. We report that many vestibular nucleus neurons exhibit systematic and significant changes in their firing rates at eccentric eye positions, consistent with the hypothesis that VN neurons encode $3 \mathrm{D}$ eye velocity during smooth-pursuit eye movements.

\section{Materials and Methods}

Two juvenile rhesus monkeys were implanted with dual eye coils and prepared for chronic recording of binocular 3D eye movements and single-unit activity. During the experiments, monkeys were placed (with their heads tilted $18^{\circ}$ nose-down relative to the stereotaxic horizontal plane) in a primate chair that was secured inside the inner frame of a vestibular turntable with two independently controlled rotational drives that could rotate animals in yaw, roll, or pitch (Acutronics, Pittsburgh, PA). Both stimulus presentation and data acquisition were controlled using the Cambridge Electronics device (CED) (model power 1401; Cambridge Electronics, Cambridge, UK) data acquisition system and custom-written scripts for the Spike2 interface (for details, see Angelaki et al., 2001). Animals followed a small target light that was back-projected onto a screen at a distance of $\sim 27 \mathrm{~cm}$ (vergence angle of $6.4^{\circ}$ ) using a laser and $x-y$ mirror galvanometer system (General Scanning, Watertown, MA).

Extracellular recordings from vestibular nuclei (VN) neurons were obtained using standard electrophysiological techniques. Neural activity was amplified, filtered $(300 \mathrm{~Hz}-6 \mathrm{kHz})$, and passed both to an audio amplifier and a BAK Electronics (Germantown, MD) dual timeamplitude window discriminator that generated acceptance pulses for the CED. Stimuli and eye movement signals were antialias filtered (200 $\mathrm{Hz}$, six-pole Bessel) and digitized by the CED at a rate of $833.33 \mathrm{~Hz}$ (16-bit resolution).

Penetrations concentrated in a relatively small area in the rostral part
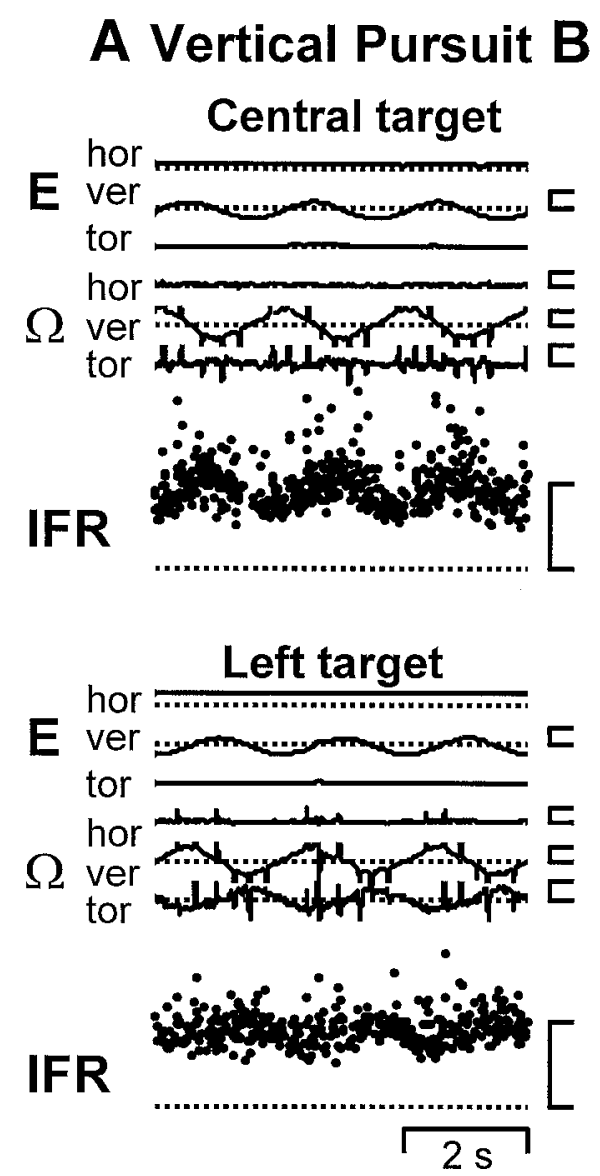

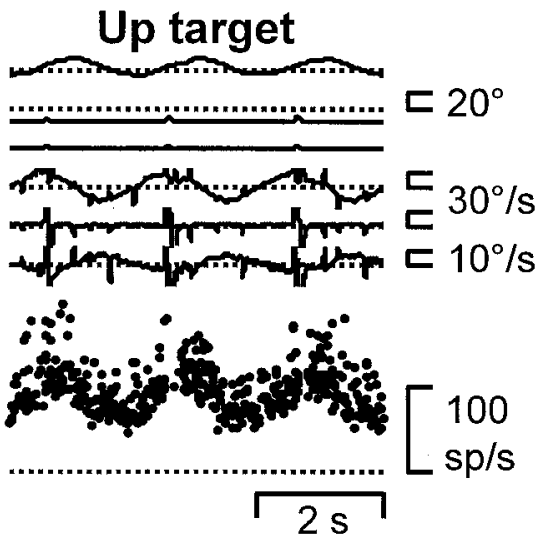

Figure 1. Responses of an eye-head neuron during vertical and horizontal pursuit $\left(0.5 \mathrm{~Hz} \pm 10^{\circ}\right)$ with the target located either straight ahead (top), to the left (for vertical pursuit; bottom left) or up (horizontal pursuit; bottom right). For each panel, the three components of eye position ( $E_{\text {hor }}$, horizontal; $E_{v e r}$, vertical; and $E_{\text {tor }}$ torsional) and the three components of the angular velocity $\left(\Omega_{\text {hor }}\right.$, horizontal; $\Omega_{\text {ver }}$ vertical; and $\Omega_{\text {tor }}$, torsional) of the right eye and IFR of the neuron are shown. Positive directions of eye movements are leftward, downward, and clockwise (relative to the animal). Vertical scale bars, $20^{\circ}$ for eye position, $30^{\circ} / \mathrm{sec}$ for horizontal-vertical, and $10^{\circ} \mathrm{sec}$ for torsional eye velocity. Note that, because of the mathematics of rotational kinematics, $\Omega_{\text {tor }}$ is not the time derivative of $\mathrm{E}_{\text {tor }}$ (Tweed and Vilis, 1987, 1990; Haslwanter, 1995). 
yaw rotation

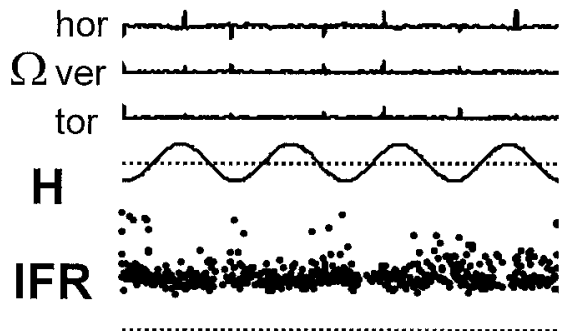

pitch rotation
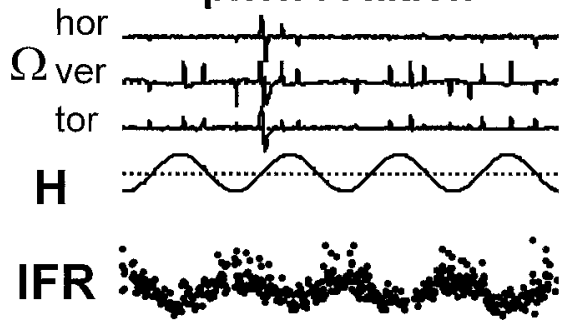

roll rotation

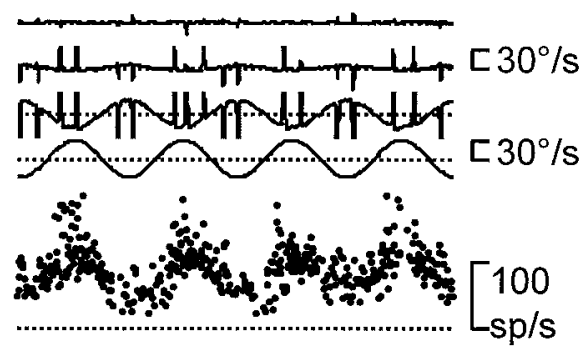

RALP rotation
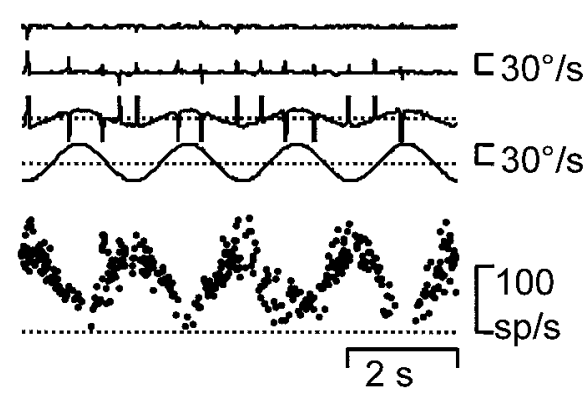

Figure 2. Responses of the same eye-head neuron as in Figure 1 during rotation about different axes $\left(0.5 \mathrm{~Hz}, \pm 10^{\circ}\right)$. The animal was viewing a head-fixed target located straight ahead during yaw, pitch, roll, or right anterior-left posterior canal plane (RALP) rotation. For each panel, the three components of eye position $\left(E_{\text {hor }}\right.$, horizontal; $E_{\text {ver, }}$ vertical; and $E_{\text {tor }}$, torsional) and the three components of the angular velocity $\left(\Omega_{\text {hor }}\right.$, horizontal; $\Omega_{\text {ver, }}$ vertical; and $\Omega_{\text {tor, }}$ torsional) of the right eye, stimulus (head velocity, $H$ ), and IFR of the neuron. Positive directions of eye and head movements are leftward, downward, and clockwise (relative to the animal). Vertical scale bars, $30 \%$ sec for horizontal-vertical-torsional eye and head velocity.

of the medial and ventrolateral $\mathrm{VN}$, extending $\sim 3 \mathrm{~mm}$ posterior and to $\sim 3 \mathrm{~mm}$ lateral from the abducens nuclei (for recording sites, see Dickman and Angelaki, 2002, their Fig. 1). Once a VN neuron was isolated, each cell was typed on the basis of its responses during horizontal and vertical smooth pursuit $\left(0.5 \mathrm{~Hz}, \pm 10^{\circ}\right)$, visually guided saccades, and 0.5 $\mathrm{Hz}\left( \pm 10^{\circ}\right)$ yaw and pitch oscillations (with the animal upright and fixating a head-fixed target). On the basis of the neural responses during these protocols, eye movement-sensitive cells were classified into one of three groups (Scudder and Fuchs, 1992), as follows. (1) Positionvestibular-pause and position-vestibular (referred to collectively as PV) neurons were characterized by sensitivities to head velocity and eye velocity in opposite directions. (2) Eye-head (EH) neurons exhibited sensitivities to head velocity during VOR suppression and to eye velocity during smooth pursuit in the same direction. (3) Burst-tonic or purely tonic (referred to collectively as BT) neurons did not modulate during either yaw or pitch VOR suppression but exhibited significant responses during either horizontal or vertical fixations and smooth-pursuit eye movements.

After cell characterization as PV, EH, or BT, animals were asked to pursue a target that moved either horizontally or vertically $(0.5 \mathrm{~Hz}$, $\pm 10^{\circ}$ ) at different eccentricities (up to $\pm 25^{\circ}$ ). Because during eccentric pursuit, the axis of eye rotation deviates from pure horizontal or vertical with a proportional increase in the torsional component, we used these protocols to examine whether neural firing rates during pursuit exhibit sensitivity to torsional eye velocity. Cell responsiveness was also tested during rotations $\left(0.5 \mathrm{~Hz}, \pm 10^{\circ}\right)$ in different head planes, including roll, right anterior-left posterior and left anterior-right posterior canal planes, and in-between axes. During all rotations, animals were rewarded for maintaining fixation on a head-fixed central target, thus suppressing changes in gaze direction (but typically not torsional eye velocity).

Neural activity was expressed as instantaneous firing rate (IFR), which was computed as the inverse of interspike interval and assigned to the middle of the interval. Eye positions were calibrated and expressed as 3D rotation vectors using straight ahead as the reference position, whereas eye angular velocity was computed as described previously (Hepp, 1990).
In both animals, Listing's plane (computed from spontaneous eye movement data in the light) was within $<2-3^{\circ}$ of the frontal (horizontal-vertical) plane. Thus, we will refer to "Listing's plane" and "horizontal-vertical plane" interchangeably.

Desaccaded neural firing rates from multiple cycles for each stimulus condition were folded into a single cycle (no averaging was performed). Only portions in which the positions of both eyes were within $\pm 1.5^{\circ}$ of the target were included in the folding for additional analyses. The neural response sensitivity and phase during rotation and pursuit were determined by fitting a sine function (first and second harmonics and a DC offset) to the overlaid data using a nonlinear least-squares algorithm based on the Levenberg-Marquardt method. Portions of the cycle in which neurons were silent were excluded from the least-squares optimization. Response gains were estimated in spikes/second per degree/second. Phase was expressed as the difference (in degrees) between peak neural activity and peak head or eye (for pursuit) velocity. Positive directions for eye and head motion were leftward, downward, and clockwise (from the animal's perspective).

The spatial tuning characteristics of the cells during pursuit eye movements in primary position were evaluated using either a cosine spatial-tuning model (ignoring variations in phase) or a spatiotemporal model (that considers both gain and phase) (Angelaki, 1991; Angelaki and Dickman, 2000; Leung et al., 2000). The latter is more general, because it provides the ability to describe neural firing rates in cases in which the response of the cell in the minimum-sensitivity direction is nonzero and response modulation phase is not identical during horizontal and vertical pursuit. Spatiotemporal tuning of cells during pursuit has been reported previously to exist in flocculus-ventral paraflocculus Purkinje cells (Leung et al., 2000). Because the majority of PV and $\mathrm{EH}$ cells exhibited different response phases for horizontal and vertical pursuit, the spatiotemporal model fits are reported here.

Neural responses during eccentric pursuit were quantified in two ways. First, linear regressions of response gain and phase as a function of gaze eccentricity during pursuit were used to characterize whether neurons changed their firing rates as a function of eye position during eccentric pursuit. If neurons exhibited $3 \mathrm{D}$ sensitivity to eye movements, a significant correlation would be expected. This regression analysis provided an intuitive, yet indirect, test of the underlying hypothesis. Thus, in addition, a mathematically more vigorous approach was used when the cumulative cycles (see above) of cell modulation for pursuit responses at all eccentricities for each cell were fit simultaneously with two models. The first (2D) model assumed that neural firing rates could be predicted using a linear combination of horizontal and vertical eye movements. The second (3D) model assumed that neural firing rates could be predicted as a linear combination of sensitivities to horizontal, vertical, and torsional eye movements. Specifically, the 2D multiple linear regression model used was of the following form:

$$
\mathrm{FRM}=r_{1}{ }^{\star} H_{o} \sin \omega t+\mathrm{k}_{1}^{\star} H_{o} \cos \omega t+r_{2}{ }^{\star} V_{o} \sin \omega t+k_{2}^{\star} V_{o} \cos \omega t,
$$

Similarly, the 3D multiple linear regression model used was of the following form:

$$
\begin{aligned}
\mathrm{FRM}=r_{1}{ }^{\star} H_{o} \sin \omega t & +k_{1}{ }^{\star} H_{o} \cos \omega t+r_{2}{ }^{\star} V_{o} \sin \omega t \\
& +k_{2}{ }^{\star} V_{o} \cos \omega t+r_{3}{ }^{\star} T_{o} \sin \omega t+k_{3}{ }^{\star} T_{o} \cos \omega t,
\end{aligned}
$$


where FRM is the firing rate modulation of the cell, $H_{o}, V_{o}$, and $T_{o}$ are peak horizontal, vertical, and torsional eye velocity, respectively, and $\omega=$ $\pi$ (at a frequency of $0.5 \mathrm{~Hz}$ ).

To compare the ability of each model to describe the gain and/or phase dependence on eye position, three measures were used. First, varianceaccounted-for (VAF) coefficients were computed as follows:

$$
\mathrm{VAF}=\{1-[\operatorname{var}(\text { model }- \text { data }) / \operatorname{var}(\text { data })]\},
$$

which provided a normalized measure of the goodness of fit. For example, $\mathrm{a} \mathrm{VAF}=0.50$ indicates that $50 \%$ of the gain or phase dependence on eye position is described by the model. Because the $2 \mathrm{D}$ and $3 \mathrm{D}$ models were associated with a different number of free parameters (four versus six, respectively), two cost indexes were computed, in addition to VAF. One was the mean square error (MSE), computed as follows:

$$
\operatorname{MSE}=\sum_{i}[M(i)-\operatorname{data}(i)]^{2} /(N-P),
$$

where data( $i)$ represents the firing rate modulations measured experimentally at each gaze position, $M(i)$ the corresponding values estimated from the model fit, $N$ the number of different target eccentricities tested, and $P$ the number of model parameters fitted. In addition, the Bayesian information criteria (BIC) measure was computed for a second measure of a cost index (Caines, 1988; Cullen et al., 1996) as follows:

$$
\mathrm{BIC}=\log \left(\frac{1}{N} \sum_{i}[M(i)-\operatorname{data}(i)]^{2}\right)+\frac{P}{2} \log \frac{\log N}{N},
$$

Whereas the VAF measure provides a goodness-of-fit criterion that is independent of the number of model parameters fitted, the MSE and BIC measures take into account the number of parameters fitted. For the 3D model to be more appropriate to describe neural firing rates during pursuit, VAF values should be larger, whereas MSE and BIC values should be lower than those for the 2D model. Because BIC and MSE exhibited comparable differences for the $2 \mathrm{D}$ and $3 \mathrm{D}$ model fits, only the VAF and BIC values obtained for the two model fits have been illustrated here.

The linear regression analysis described by Equation 1 provided a horizontal, vertical, and torsional gain and phase for each cell. For each of these components, these gain and phase values were computed from the respective $r$ and $k$ coefficients, as follows:

$$
\text { gain } S=\sqrt{r^{2}+k^{2}} \text { and phase } \theta=\arctan (k / r) .
$$

Because phases were generally not identical for the horizontal, vertical, and torsional components, the same spatiotemporal model as used to compute the maximum-sensitivity vector to pursuit through primary position was also used to calculate the maximum-sensitivity vector in $3 \mathrm{D}$ (Angelaki, 1991). Briefly, each direction in space was represented in spherical coordinates, using two angles, the azimuth in Listing's plane, $\alpha$ (defined as the angle $0^{\circ}<\alpha<360^{\circ}$ that the projection of the 3D vector onto Listing's plane forms with the vertical axis), and the elevation from Listing's plane, $\beta\left(-90^{\circ}<\beta<90^{\circ}\right)$. If $S_{h}, S_{v}, S_{t}$, and $\theta_{h}, \theta_{v}, \theta_{t}$ are the respective gain and phase for the horizontal, vertical, and torsional components of the firing of the cell (computed from Eqs. 1,2), the sensitivity and phase along any arbitrary direction defined by its azimuth, $\alpha$, and elevation, $\beta$, are given by the following equations (Angelaki, 1991):

$S(\alpha, \beta)$

$=\sqrt{S_{L}^{2}(\alpha) \cos ^{2}(\beta)+S_{t}^{2} \sin ^{2}(\beta)+S_{L}(\alpha) S_{t} \sin (2 \beta) \cos \left(\theta_{t}-\phi_{L}(\alpha)\right)}$,

and

$$
\begin{aligned}
\sin \phi & =\frac{S_{v} \sin \theta_{v} \cos \alpha \cos \beta+S_{h} \sin \theta_{h} \sin \alpha \cos \beta+S_{t} \sin \theta_{t} \sin \beta}{S} \\
\cos \phi & =\frac{S_{v} \cos \theta_{v} \cos \alpha \cos \beta+S_{h} \cos \theta_{h} \sin \alpha \cos \beta+S_{t} \cos \theta_{t} \sin \beta}{S}
\end{aligned}
$$

where $S_{L}(\alpha)$ and $\phi_{L}(\alpha)$ represent the gain and phase of the projection onto Listing's plane and are calculated from the following:

$$
S_{L}(\alpha)=\sqrt{S_{v}{ }^{2} \cos ^{2}(\alpha)+S_{h}{ }^{2} \sin ^{2}(\alpha)+S_{v} S_{h} \sin (2 \alpha) \cos \left(\theta_{v}-\theta_{h}\right)}
$$

and

$$
\begin{aligned}
\sin \phi_{L} & =\frac{S_{v} \sin \theta_{v} \cos \alpha+S_{h} \sin \theta_{h} \sin \alpha}{S_{L}} \\
\cos \phi_{L} & =\frac{S_{\nu} \cos \theta_{v} \cos \alpha+S_{h} \cos \theta_{h} \sin \alpha}{S_{L}} .
\end{aligned}
$$

The amplitude of the maximum-sensitivity vector was computed as the maximum of $S(\alpha, \beta)$ (Eq. 3). The corresponding values of $\alpha$ and $\beta$ gave its spatial orientation, whereas its phase could be calculated from Equation 4, with the azimuth and elevation values $\alpha$ and $\beta$ corresponding to those of the maximum-sensitivity vector. The distributions of neural response vectors have been expressed in a right-handed head-fixed coordinate system relative to a fixed $18^{\circ}$ nose-down head position, which aligned the horizontal canal and utricle approximately with the horizontal rotation plane. For simplicity, vectors have been drawn as if cell activity was recorded in the left side of the brain (by reversing the signs of the $z$ - and $x$-direction cosines of cells recorded in the right VN). Because VOR responses were collected during fixation of a head-fixed target, a condition in which horizontal-vertical but not torsional slow-phase eyevelocity modulations are canceled, $3 \mathrm{D}$ vector distributions have not been illustrated for rotation.

\section{Results}

Responses to horizontal and vertical pursuit and rotation in multiple planes were recorded in 100 eye movement-related neurons in the vicinity of the rostral medial vestibular nuclei of two animals (for recording sites, see Dickman and Angelaki, 2002, their Fig. 1). On the basis of their firing activities during fixations, smooth pursuit, and rotational motion while fixating a headfixed target, neurons were classified into three groups. These included eye-head ( $\mathrm{EH} ; n=50$ ), position-vestibular-pause or position-vestibular (referred to collectively as PV; $n=41$ ), and burst-tonic or tonic (BT; $n=9$ ) cells (Keller and Kamath, 1975; Tomlinson and Robinson, 1984; Scudder and Fuchs, 1992; Cullen and McCrea, 1993; Lisberger et al., 1994). For example, "vertical" EH cells exhibited little modulation during pursuit of a target moving horizontally through the primary position (Fig. 1, top right traces) and during yaw VOR suppression (Fig. 2, top left traces). In contrast, the cell clearly modulated during pursuit of a vertically moving target, increasing its firing rate approximately in phase with upward (negative) eye velocity (Fig. 1, top left traces) and during pitch oscillations in the absence of gaze changes (Fig. 2, bottom left traces). During pitch oscillations, peak firing rate was approximately in phase with upward (negative) head velocity, a fact that would identify this neuron clearly as a vertical EH cell.

When the animal was asked to pursue moving targets that did not pass through the primary position, the directional preference of the cell changed (Fig. 1, bottom traces). Specifically, the vertical pursuit sensitivity of the cell was reduced when the animal was following a target that moved up-down but was located to the left of primary position. In contrast, the horizontal pursuit sensitivity of the cell was increased when the animal was following a target that was displaced upward relative to primary eye position. Thus, the primary sensitivity of the cell changed from vertical to horizontal pursuit, as the target moved from central to eccentric (tertiary) positions.

Examination of the eye movement components during pur- 


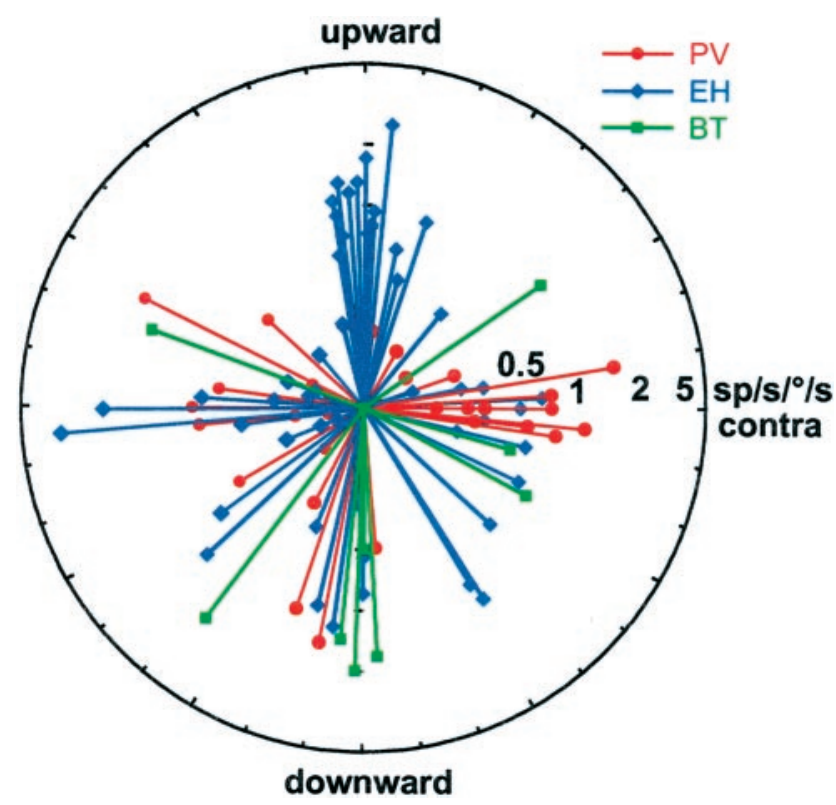

Figure 3. Spatial distribution of pursuit response vectors for 100 neurons tested during horizontal-vertical pursuit through primary position. Different symbols represent PV (red cir(les), EH (blue diamonds), and BT ( green squares) cells. Dotted circular lines illustrate concentric circles at a constant sensitivity $(2,1$, and 0.5 spikes $/$ sec per $\%$ sec). Dotted radial lines represent $30^{\circ}$ increments in spatial alignment.

suit for targets at different eccentricities suggests an explanation for these differences in responsiveness. During pursuit through primary position (central target), eye velocity remained in Listing's plane, with only vertical and horizontal eye movement components and little torsional eye velocity (Fig. 1, top traces). In contrast, during pursuit in eccentric eye positions, the velocity axis of the eye tipped out of the horizontal-vertical plane with the addition of a significant component along the torsional axis (half-angle rule) (Tweed and Vilis, 1987, 1990; Haslwanter et al., 1991; Tweed et al., 1992). Specifically, during vertical pursuit of horizontally eccentric targets, the elicited eye velocity was not purely vertical. Similarly, during horizontal pursuit of vertically eccentric targets, the eye velocity produced was not purely horizontal (Fig. 1, bottom). Rather, torsional eye velocity components were clearly present during eccentric pursuit for both cases.

The apparent reversal in the pursuit preference of the cell could simply reflect an underlying sensitivity to torsional eye velocity. For example, if the cell shown in Figure 1 had a preference for a combination of upward (negative vertical) and counterclockwise (negative torsional) eye velocity, the cell would modulate in phase with negative torsional eye velocity during horizontal pursuit of an upward-eccentric target. Similarly, because positive torsional velocity accompanies negative vertical velocity during vertical pursuit of a leftward-eccentric target, the vertical and torsional sensitivities of the cell would oppose each other, resulting in a reduction in the modulation amplitude of the cell, as observed experimentally. The hypothesized sensitivity of this neuron to counterclockwise (negative) torsional velocity would also be consistent with its response modulation during roll and combined roll-pitch oscillations (Fig. 2, right traces).

\section{Pursuit through primary position}

To describe quantitatively the preferences of the neurons to $2 \mathrm{D}$ eye velocity during the more traditionally used tasks of horizontal and vertical pursuit through primary position, response sensitivity and phase were fitted using a spatiotemporal extension of the cosine spatial-tuning model (Angelaki, 1991; Leung et al., 2000).
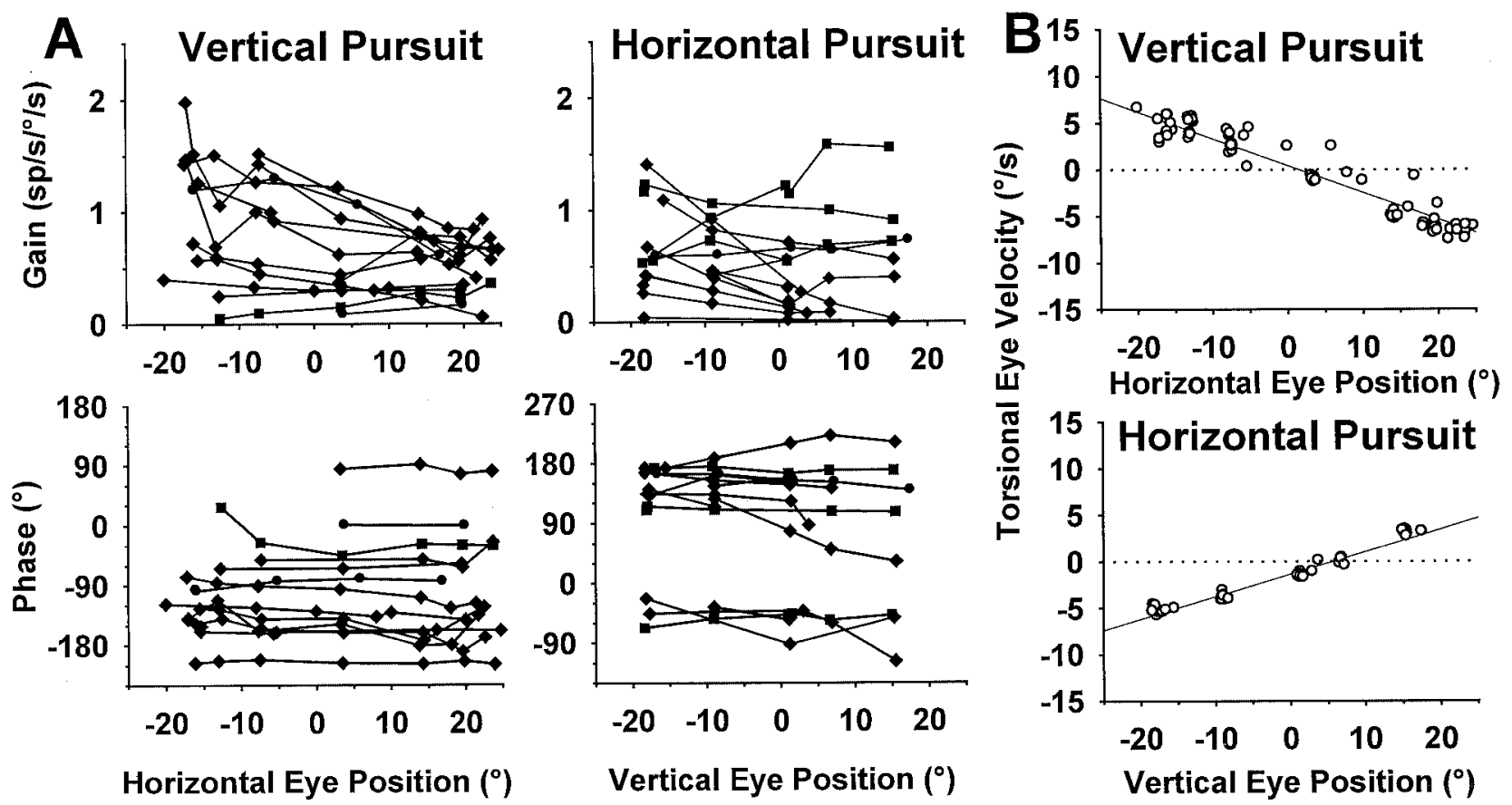

Figure 4. Dependence of $(A)$ neural firing rates and $(B)$ torsional eye velocity on horizontal (left) and vertical (right) gaze eccentricity during vertical and horizontal pursuit, respectively. Data from $16 \mathrm{PV}$ (red circles), EH (blue diamonds), and BT ( green squares) cells that exhibited a significant dependence of gain and/or phase on eye position are shown. Data in $B$ were recorded simultaneously with the neural responses in $A$. Torsional eye velocity is shown above the dotted zero-line when positive torsion is elicited simultaneously with positive vertical (downward) or positive horizontal (leftward) eye velocity and below the dotted zero-line otherwise. The small nonzero torsional values at zero eye position (straight-ahead gaze) reflect the small difference between straight ahead and primary position (see Materials and Methods). Neural response phase was expressed relative to downward and ipsilateral eye velocity for vertical and horizontal pursuit, respectively. A phase of $0^{\circ}\left(-90^{\circ}\right)$ corresponded to a response in phase with downward eye velocity (position) during vertical pursuit and ipsilateral eye velocity (position) during horizontal pursuit. 
The distribution of maximum sensitivity vectors in the horizontal-vertical plane is shown in Figure 3. The majority of vectors were directed within $\pm 30^{\circ}$ of one of the cardinal axes, indicating a preference for either horizontal or vertical eye movements. Only 2 BT, 5 PV, and 7 EH neurons (14\% of the total cells tested) were equally sensitive to horizontal and vertical eye movements. As seen from Figure 3, most vertical EH cells sampled preferred upward eye movements. In contrast, vertical PV and BT neurons preferred almost exclusively downward eye movements.

\section{Pursuit in eccentric eye positions}

Of the 100 neurons characterized, 31 were examined during pursuit at eccentric positions. Of these, approximately half (11 of 19 $\mathrm{EH}, 2$ of 3 PVP, and 3 of 9 BT) of the neurons exhibited a significant $(p<0.05)$ dependence of their response gain and/or phase on target eccentricity. The pursuit gain and phase as a function of eccentricity for these 16 cells have been plotted in Figure $4 \mathrm{~A}$. As already illustrated in the example in Figure 1, a torsional eye velocity component developed during pursuit at tertiary positions. The magnitude of torsional eye velocity increased linearly with gaze eccentricity, and its phase reversed for upward-downward or leftward-rightward targets (Fig. 4B). Thus, a cell that was sensitive to torsional (in addition to horizontal or vertical) eye velocity would be expected to change its firing rate modulation during eccentric pursuit (Fig. 4A). Most (10 of $11 \mathrm{EH}$, one PVP, and one BT) of these cells with significant gaze direction dependence were classified as vertical neurons during pursuit through primary position. In fact, 10 of a total of 13 vertical EH neurons tested with eccentric pursuit changed their modulation significantly during pursuit at eccentric eye positions. Like the neuron whose responses are illustrated in Figure 1, most EH neurons exhibited sensitivity to negative torsional eye velocity [thus, peak firing rates decreased with increasing positive horizontal (leftward) and vertical (downward) eye position] (Fig. 4A).

The regression analyses presented above demonstrated that in many neurons, there was a significant dependence of their firing rates during pursuit on eye position. This is necessary (but not sufficient) to demonstrate neural sensitivities to $3 \mathrm{D}$ eye movements. Thus, to test quantitatively the hypothesis that the gazedependent behavior during pursuit arose because of a sensitivity to torsional eye movements, we compared the fits between a 3D multiple linear regression model (six parameters; Eq. 1b) and a more traditionally used 2D (horizontal-vertical, four parameters; Eq. 1a) model. Each model was fit simultaneously to all available cumulative cycles of horizontal-vertical pursuit at different target eccentricities for each cell. As shown in Figure $5 A$, for all cells, the 3D model provided fits with higher VAF values, and in most cells (26 of 31 ), smaller BIC values than the $2 \mathrm{D}$ model. The maximum-sensitivity vectors of these neurons in $3 \mathrm{D}$ were then computed from the gain and phase values calculated from the multiple linear regression results using a spatiotemporal model (Angelaki, 1991). The amplitude and absolute value of elevation from the horizontal-vertical plane (abscissa) are illustrated in Figure $5 B$. Nineteen of the 31 neurons had preferred sensitivity vectors $>30^{\circ}$ away from the horizontal-vertical plane. Most of the cells whose preferred vectors were $>30^{\circ}$ outside the horizontal-vertical plane also exhibited a significant dependence of pursuit response gain and/or phase on target eccentricity (Fig. $5 B$, filled symbols). In contrast, most cells whose gain and phase dependence on eye position was not significant (Fig. 5B, open symbols) had pursuit sensitivity vectors within a $30^{\circ}$ range from the horizontal-vertical plane.

The spatial distribution of preferred pursuit $3 \mathrm{D}$ vectors have

\section{A Comparison of goodness of fit}
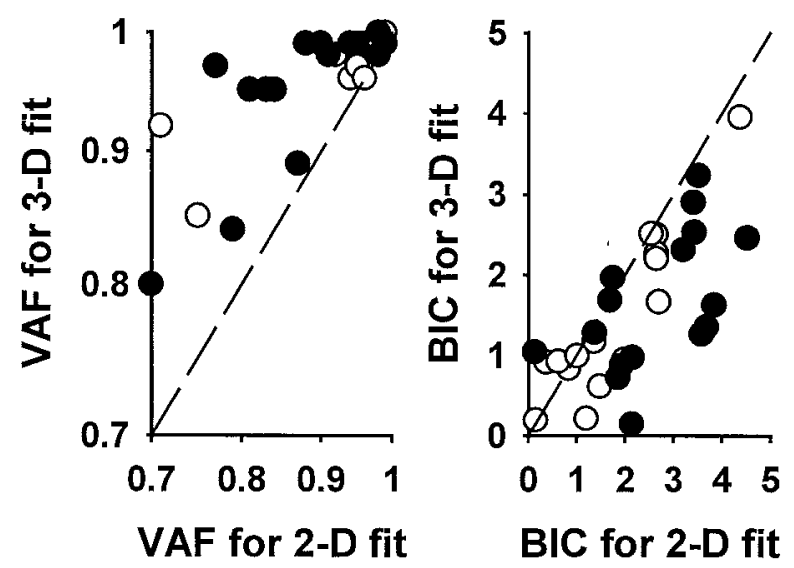

\section{$B$ 3D vector distribution for pursuit}

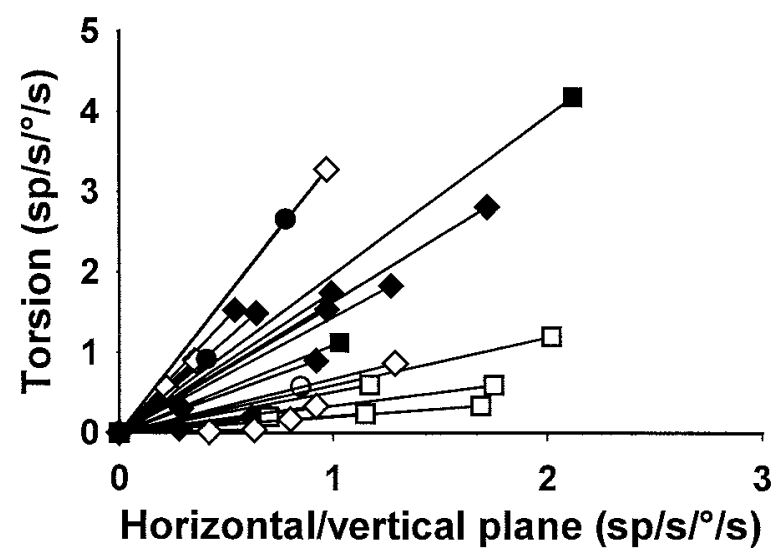

Figure 5. Quantification of the torsional eye movement sensitivity of neurons. A, Comparison of two goodness-of-fit criteria, the variance-accounted-for (VAF) and the Bayesian information criteria (BIC), obtained for the traditional 2D (horizontal-vertical) versus 3D model fits. VAF and BIC values for the 3D model fall above or below (respectively) the corresponding values obtained from the 2D model. Filled symbols correspond to fits for neurons with significant target position dependence (Fig. 4A). Open symbols are used for cells whose regressions were not significant. $B$, Spatial plots of the computed 3D eye movement sensitivity vector for 31 cells tested during eccentric pursuit. Each line corresponds to a single cell, with its length representing the sensitivity of the cell to pursuit and its orientation corresponding to the absolute value of the elevation angle, $\beta$, out of Listing's plane toward the torsional axis (Eq. 3). Filled symbols were used for the same PV (red circles), EH (blue diamonds), and BT ( green squares) cells as those plotted in Figure 4. Open symbols were used for the remaining cells whose gain and phase dependence on target eccentricity was not rendered significant according to linear regressions similar to those in Figure 4.

been plotted as projections onto the three cardinal head planes in Figure 6. The vector distribution appeared scattered, with no clear clustering along the axes of the semicircular canals (Fig. 6, thick red, green, and cyan lines).

\section{Discussion}

During pursuit of eccentrically placed targets, eye position and the derivative of eye position (which is not equal to angular eye velocity unless the eye is in primary position) remain confined to Listing's plane. In contrast, the axis about which the eye rotates (i.e., direction of eye angular velocity) tips out of this plane by half the gaze angle (Tweed and Vilis, 1987; Haslwanter et al., 1991; Tweed et al., 1992). Thus, during pursuit in eccentric positions, 


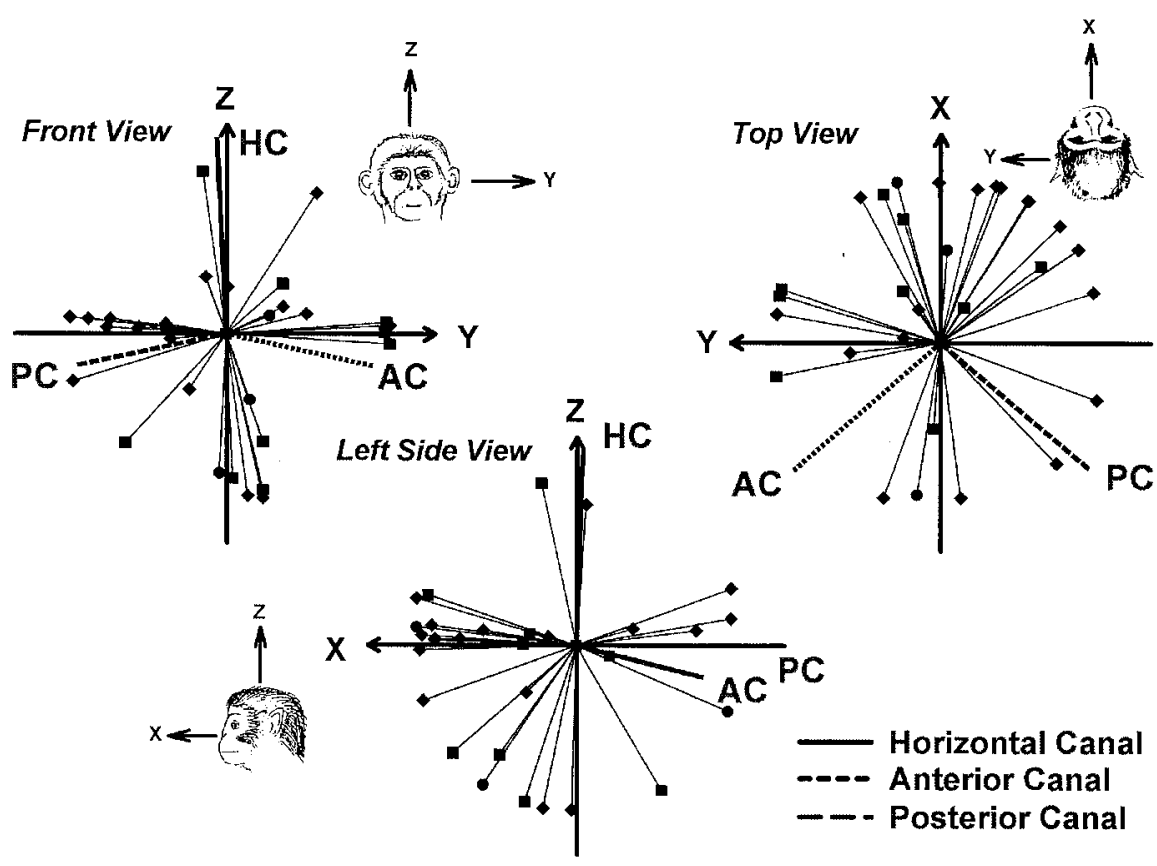

Figure 6. 3D spatial distribution of pursuit preferred directions (unit vectors) for 31 cells. The mean vector orientations for the ipsilateral (left) horizontal canal (HC), anterior canal ( $A C$ ), and posterior canal $(P C$ afferents have been plotted with heavy, long lines (magenta, green, and cyan lines, respectively) (data from Dickman et al., 2002). As rotations are represented using the right-hand rule, a preferred sensitivity for ipsilateral (leftward) rotation is represented as a vector aligned with the positive z-axis. Similarly, sensitivity to a downward rotation is illustrated as a vector aligned with the positive $y$-axis. Finally, a clockwise rotation (i.e., rotation toward right ear down from upright) is represented as a vector aligned with the positive $x$-axis. Red circles illustrate $\mathrm{PV}$; blue diamonds, EH responses; and green squares, BT cells.

the velocity of the eye is not purely horizontal-vertical but rather exhibits significant torsional components. We have shown here that the majority of vertical VN eye movement neurons exhibited significant changes in their firing rates that paralleled the monotonic increase in torsional eye velocity as a function of eye eccentricity. Multiple linear regression analyses of cell responsiveness during pursuit at multiple eye orientations revealed a significant sensitivity to the torsional component of the eye movement for the majority of vertical and many horizontal VN cells. Thus, the preferred directions of VN cells during pursuit were characterized by a $3 \mathrm{D}$ distribution that was not restricted to the horizontal-vertical eye movement plane, as one might have expected on the basis of recent hypotheses about the neural substrates for visually driven eye movements like pursuit and saccades (Quaia and Optican, 1998; Raphan, 1998; Demer et al., 2000). These observations not only have important implications about the premotor coordinate system for pursuit but also provide insight into the controversy regarding eye torsion control (Miller, 1989; Tweed et al., 1994; Tweed, 1997; Quaia and Optican, 1998; Smith and Crawford, 1998; Demer et al., 2000; Misslisch and Tweed, 2001).

\section{Previous studies of vertical eye movement neurons}

Previously, vertical eye movement neurons in primates have been characterized in the medial longitudinal fasciculus (King et al., 1976; Pola and Robinson, 1978) and the superior, medial VN, and $y$-group (Tomlinson and Robinson, 1984; Partsalis et al., 1995; Zhang et al., 1995). However, to the best of our knowledge, the responses of vertical neurons have not been tested previously during pursuit in eccentric eye positions. On the basis of neuroanatomic data, the excitatory posterior canal pathway to the ocular motoneurons is thought to be through the medial $\mathrm{VN}$ and the medial longitudinal fasciculus (Highstein and McCrea, 1988). Both downward and upward eye movement-sensitive cells projecting to either the trochlear and/or oculomotor nuclei should be encountered in the medial VN (McCrea et al., 1987). In the present study, the majority of vertical $\mathrm{EH}$ cells increased their firing rates with upward eye movements, and the majority of vertical PV neurons increased their firing rates with downward eye movements (Fig. 3). Both of these groups of neurons increased their firing rates during upward head movements, a fact that is consistent with a vestibular signal arising from the posterior canal.

\section{Implications for the coordinate system} for premotor coding of pursuit

It has long been known that visual (optokinetic) and vestibular signals share a common, SCC-based coordinate system in the brainstem and cerebellum of lateral-eyed, afoveate species (Graf et al., 1988; Tan et al., 1993; Wylie and Frost, 1993; van der Steen et al., 1994). Such a common coordinate system would be important for effective and coordinated gaze stability during natural activities, in which optokinetic and vestibular reflexes operate in synergy to keep eye orientation stable in space. Premotor coding of visuomotor signals in SCC coordinates was by no means surprising, because both optokinetic and vestibular reflexes operate in $3 \mathrm{D}$, with torsional eye movements representing a clear component of both optokinetic nystagmus and the VOR (Collewijn and Noorduin, 1972; Cheung and Howard, 1991; Morrow and Sharpe, 1993; Thilo et al., 1999).

Whether a similar organization also underlies pursuit-VOR interactions in foveate species was unknown. Because animals cannot pursue "torsionally," one could argue that the neural coding of pursuit is not $3 \mathrm{D}$, as in the VOR, but rather $2 \mathrm{D}$, like saccadic eye movements. Because both visual sensory and oculomotor output signals are $2 \mathrm{D}$ and because extraocular muscle pulleys could implement the half-angle rule of both pursuit and saccades (Quaia and Optican, 1998), neural firing rates could always encode pursuit eye movements in Listing's plane throughout the sensorimotor and motor pathways. However, because pursuit and the VOR are thought to share common premotor neurons (Scudder and Fuchs, 1992; Cullen and McCrea, 1993), a common coordinate system for both behaviors seems plausible. In fact, the presence of strong torsional nystagmus during vertical pursuit in some patients with cerebellar deficits has suggested that premotor pursuit signals might be coded in an SCC coordinate frame (FitzGibbon et al., 1996). The present results, demonstrating that vertical neurons exhibit pursuit sensitivities with clear torsional components and have preferred vectors lying outside Listing's plane, would support a $3 \mathrm{D}$ coordinate system for the premotor coding of pursuit eye movements. The preferred pursuit directions were not, however, observed to exhibit a clear clustering along the SCC axes. However, because VOR responses were recorded only during fixation of a foveal head-fixed target, a direct comparison between 3D pursuit and VOR vectors for each cell was not possible. Thus, although the observation of $3 \mathrm{D}$ eye move- 
ment sensitivity during pursuit would seem to be in line with a hypothesis of a similar coordinate system for pursuit and the VOR, a direct comparison is needed before a conclusion for or against a common coordinate frame is reached.

\section{Premotor neurons code for 3D angular velocity rather than the $2 \mathrm{D}$ derivative of eye orientation: implications for downstream processing}

The present results may also provide insight into the controversial issue regarding the origin of the half-angle rule for the generation of eye movements in Listing's plane. Specifically, if premotor neurons encode $3 \mathrm{D}$ angular velocity and because $3 \mathrm{D}$ eye orientation cannot be mathematically computed as the integral of angular eye velocity, a multiplicative velocity-to-position neural integration has been proposed (Tweed and Vilis, 1987, 1990). Alternatively, if the 2D derivative of eye orientation is the signal neurally encoded during visually guided eye movements such as saccades and pursuit, a linear velocity-to-position integrator and extraocular muscles with appropriately placed pulleys are sufficient to generate visually guided eye movements in Listing's plane (Quaia and Optican, 1998).

Previously, 3D angular velocity signals were never identified in the burst activity of premotor cells during saccadic eye movements (van Opstal et al., 1991, 1996; Hepp et al., 1993, 1999; Klier et al., 2001; Scherberger et al., 2001), providing evidence for the second hypothesis (Quaia and Optican, 1998; Demer et al., 2000). However, the present results demonstrate that, contrary to saccades, 3D angular velocity is encoded in the premotor pathway for pursuit eye movements. Under the assumption that the 2D derivative of eye position (and not 3D angular velocity) is in fact encoded in the burst activities throughout the premotor pathways for saccades (which still remains uncertain) (but see Hepp et al., 1999; Scherberger et al., 2001), a difference between the premotor coding of pursuit and saccades might exist.

Specifically, it is possible that the 3D eye velocity signals encoded by $\mathrm{VN}$ neurons during pursuit are specific to the pursuit pathway, whereas burst neural activities during saccades always encode eye movements in $2 \mathrm{D}$. In this case, 3D angular velocity signals during pursuit would exist in premotor pathways only to match the coordinates of the VOR. Thus, it remains possible that premotor $3 \mathrm{D}$ eye movement signals during both pursuit and the VOR are transformed downstream into the 2D derivative of eye orientation. Like saccades, VOR and pursuit signals could then be processed with a linear neural integrator and an eye plant with pulleys (Smith and Crawford, 1998). In fact, recent functional magnetic resonance imaging studies have reported that both pulley location and their anteroposterior shift with horizontal gaze are consistent with a mechanical implementation of the halfangle rule (Demer et al., 2000; Kono et al., 2002). However, the answers to these questions remain elusive until single-unit studies are performed to investigate how motoneurons and premotor neurons encode the 3D aspects of different types of eye movement, including a direct comparison between saccades, pursuit, and the VOR. Only then could a better understanding of their 3D organization be gained.

\section{References}

Angelaki DE (1991) Dynamic polarization vector of spatially tuned neurons. IEEE Trans Biomed Eng 38:1053-1060.

Angelaki DE, Dickman JD (2000) Spatiotemporal processing of linear acceleration: primary afferent and central vestibular neuron responses. J Neurophysiol 84:2113-2132.

Angelaki DE, Green AM, Dickman JD (2001) Differential sensorimotor processing of vestibulo-ocular signals during rotation and translation. J Neurosci 21:3968-3985.

Caines PE (1988) Linear stochastic systems. Toronto: Wiley.

Cannon SC, Robinson DA (1987) Loss of the neural integrator of the oculomotor system from brain stem lesions in monkey. J Neurophysiol 57:1383-1409.

Cheung BS, Howard IP (1991) Optokinetic torsion: dynamics and relation to circularvection. Vision Res 31:1327-1335

Collewijn H, Noorduin H (1972) Vertical and torsional optokinetic eye movements in the rabbit. Pflügers Arch 332:87-95.

Cullen KE, McCrea RA (1993) Firing behaviour of brain stem neurons during voluntary cancellation of the horizontal vestibuloocular reflex. I. Secondary vestibular neurons. J Neurophysiol 70:828-843.

Cullen KE, Rey CD, Guitton D, Galiana HL (1996) The use of system identification techniques in the analysis of oculomotor burst neuron spike train dynamics. J Comput Neurosci 3:347-368.

Demer JL, Oh SY, Poukens V (2000) Evidence for active control of rectus extraocular muscle pulleys. Ophthalmol Vis Sci 41:1280-1290.

Dickman JD, Angelaki DE (2002) Vestibular convergence patterns in vestibular nuclei neurons of alert primates. J Neurophysiol 88:3518-3533.

FitzGibbon EJ, Calvert PC, Dieterich M, Brandt T, Zee DS (1996) Torsional nystagmus during vertical pursuit. J Neuroophthalmol 16:79-90.

Graf W, Simpson JI, Leonard CS (1988) Spatial organization of visual messages of the rabbit's cerebellar flocculus. II. Complex and simple spike responses of Purkinje cells. J Neurophysiol 50:2091-2121.

Haslwanter T (1995) Mathematics of three-dimensional eye rotations. Vision Res 35:1727-1739.

Haslwanter T, Straumann D, Hepp K, Hess BJ, Henn V (1991) Smooth pursuit eye movements obey Listing's law in the monkey. Exp Brain Res $87: 470-472$.

Haslwanter T, Straumann D, Hess BJ, Henn V (1992) Static roll and pitch in the monkey: shift and rotation of Listing's plane. Vision Res 32:1341-1348.

Helmholtz H (1867) Handbuch der physiologischen Optik, Ed 1. Hamburg: Voss.

Hepp K (1990) On Listing's law. Commun Math Phys 132:285-292.

Hepp K, van Opstal AJ, Straumann D, Hess BJM, Henn V (1993) Monkey superior colliculus represents rapid eye movements in a two-dimensional motor map. J Neurophysiol 69:965-979.

Hepp K, Cabungcal JH, Duersteler M, Hess BJM, Scherberger H, Straumann D, Suzuki Y, van Opstal J, Henn V (1999) 3D structure of the reticular saccade generator. Soc Neurosci Abstr 25:661.6.

Highstein SM, McCrea RA (1988) The anatomy of the vestibular nuclei. Rev Oculomot Res 2:177-202.

Keller EL, Kamath BY (1975) Characteristics of head rotation and eye movement-related neurons in alert monkey vestibular nucleus. Brain Res 100:182-187.

King WM, Lisberger SG, Fuchs AF (1976) Responses of fibers in medial longitudinal fasciculus (MLF) of alert monkeys during horizontal and vertical conjugate eye movements evoked by vestibular or visual stimuli. J Neurophysiol 39:1135-1149.

Klier EM, Wang H, Crawford JD (2001) The superior colliculus encodes gaze commands in retinal coordinates. Nat Neurosci 4: 6:627-632.

Kono R, Clark RA, Demer JL (2002) Active pulleys: magnetic resonance imaging of rectus muscle paths in tertiary gazes. Ophthalmol Vis Sci 43: 7:2179-2188

Leung H-C, Suh M, Kettner RE (2000) Cerebellar flocculus and paraflocculus Purkinje cell activity during circular pursuit in monkey. J Neurophysiol 83:13-30.

Lisberger SG, Pavelko TA, Broussard DM (1994) Responses during eye movements of brain stem neurons that receive monosynaptic inhibition from the flocculus and ventral paraflocculus in monkeys. J Neurophysiol 72:909-927.

McCrea RA, Strassman A, May E, Highstein SM (1987) Anatomical and physiological characteristics of vestibular neurons mediating the vertical vestibulo-ocular reflexes of the squirrel monkey. J Comp Neurol 264:571-594.

Miller JM (1989) Functional anatomy of normal human rectus muscles. Vision Res 29:223-240.

Misslisch H, Tweed D (2001) Neural and mechanical factors in eye control. J Neurophysiol 86:1877-1883.

Morrow MJ, Sharpe JA (1993) The effects of head and trunk position on 
torsional vestibular and optokinetic eye movements in humans. Exp Brain Res 95:144-150.

Partsalis AM, Zhang Y, Highstein SM (1995) Dorsal Y group in the squirrel monkey. I. Neuronal responses during rapid and long-term modifications of the vertical VOR. J Neurophysiol 73: 2:615-631.

Pola J, Robinson DA (1978) Oculomotor signals in medial longitudinal fasciculus of the monkey. J Neurophysiol 41:245-259.

Quaia C, Optican LM (1998) Commutative saccadic generator is sufficient to control a 3D ocular plant with pulleys. J Neurophysiol 79:3197-3215.

Raphan T (1998) Modeling control of eye orientation in three dimensions. I. Role of muscle pulleys in determining saccadic trajectory. J Neurophysiol 79:2653-2667.

Robinson DA (1981) The use of control systems analysis in the neurophysiology of eye movements. Annu Rev Neurosci 4:463-503.

Scherberger H, Cabungcal J-H, Hepp K, Suzuki Y, Straumann D, Henn V (2001) Ocular counterroll modulates the preferred direction of saccaderelated pontine burst neurons in the monkey. J Neurophysiol 86:935-949.

Schnabolk C, Raphan T (1994) Modelling three-dimensional velocity to position transformation in oculomotor control. J Neurophysiol 71:623-638.

Scudder CA, Fuchs AF (1992) Physiological and behavioral identification of vestibular nucleus neurons mediating the horizontal vestibuloocular reflex in trained rhesus monkeys. J Neurophysiol 68:244-264.

Skavenski AA, Robinson DA (1973) Role of abducens neurons in the vestibuloocular reflex. J Neurophysiol 36:724-738.

Smith MA, Crawford JD (1998) Neural control of rotational kinematics within realistic vestibuloocular coordinate systems. J Neurophysiol 80:2295-2315.

Tan HS, van der Steen J, Simpson JI, Collewijn H (1993) Three-dimensional organization of optokinetic responses in the rabbit. J Neurophysiol 69:303-317.

Thilo KV, Probst T, Bronstein AM, Ito Y, Gresty MA (1999) Torsional eye movements are facilitated during perception of self-motion. Exp Brain Res 126:495-500.
Tomlinson RD, Robinson DA (1984) Signals in vestibular nucleus mediating vertical eye movements in the monkey. J Neurophysiol 51:1121-1135.

Tweed D (1997) Velocity-to-position transformation in the VOR and the saccadic system. In: Three-dimensional kinematics of eye, head, and limb movements (Fetter M, Haslwanter T, Misslisch H, Tweed D, Eds), pp 375-386. Amsterdam: Harwood.

Tweed D, Vilis T (1987) Implications of rotational kinematics for the oculomotor system in three dimensions. J Neurophysiol 58:823-849.

Tweed D, Vilis T (1990) Geometric relations of eye position and velocity vectors during saccades. Vision Res 30: 1:111-127.

Tweed D, Fetter M, Andreadaki S, Koenig E, Dichgans J (1992) Threedimensional properties of human pursuit eye movements. Vision Res 32: $1225-1238$

Tweed D, Misslisch H, Fetter M (1994) Testing models of the oculomotor velocity-to-position transformation. J Neurophysiol 72:1425-1429.

van der Steen J, Simpson JI, Tan J (1994) Functional and anatomic organization of three-dimensional eye movements in rabbit cerebellar flocculus. J Neurophysiol 72:31-46.

van Opstal AJ, Hepp K, Hess BJM, Straumann D, Henn V (1991) Tworather than three-dimensional representation of saccades in monkey superior colliculus. Science 252:1313-1315.

van Opstal AJ, Hepp K, Suzuki Y, Henn V (1996) Role of the monkey nucleus reticularis tegmenti pontis in the stabilization of Listing's plane. J Neurosci 16:7284-7296.

Wylie DR, Frost BJ (1993) Responses of pigeon vestibulocerebellum neurons to optokinetic stimulation. II. The 3-dimensional reference frame of rotation neurons in the flocculus. J Neurophysiol 70:2647-2659.

Zhang Y, Partsalis AM, Highstein SM (1995) Properties of superior vestibular nucleus flocculus target neurons in the squirrel monkey. I. General properties in comparison with flocculus projecting neurons. J Neurophysiol 73:2261-2278. 